\title{
Research on the Status and Trend of Contemporary Plant Landscape Construction
}

\author{
Yang Cheng \\ Jingzhou Institute of Technology, Jingzhou, Hubei Province, 434020
}

Keywords: Plant Landscaping; Current Situation; Development Trend

\begin{abstract}
Garden art, the crystallization of Chinese history and culture for thousands of years, has achieved high artistic attainments through the historical precipitation and the baptism of the years. Plant landscape construction, as an important part of the garden art, has become an important means of urban artificial landscape because of beautifying the environment to give visual, smell and other sensory enjoyment to the people. This paper first briefly summarizes the connotation and function of plant landscaping, then analyzes the present situation and probes into the development trend of it in China, hoping to provide some valuable reference to the relevant personnel.
\end{abstract}

\section{Introduction}

China, a country with a history of thousands of years of civilization, has accumulated profound material culture and spiritual culture with the historical development. As an important component of Chinese culture, having appeared as early as the Shang Dynasty, plant landscape construction has been widely praised in the world at present and has become an important symbol of contemporary Chinese traditional culture at the same time for its strong artistic and aesthetic characteristics. In the contemporary landscape planning, plant landscaping conforms to the aesthetic needs of people, and reflects the ecological concept, offering plant landscaping a broad development prospects.

\section{Overview of Plant Landscaping}

Nature has a very rich variety of plants which have their value of existence. People achieve the purpose of landscape construction with the help of natural scenery in the planning and design of gardens ${ }^{[1]}$. In short, plant landscaping mainly refers to the use of various plants to create scenery where the key is to produce a series of changes through human actions, so that plants can achieve the desired results. In the process of plant landscape building, the relevant personnel should design the plants into an artistic and aesthetic landscape according to the different characteristics and appearance of the plants.

A plenty of plants are included in the gardens, and different shapes of plants, flowers and fruits provide people with different aesthetic enjoyment. The first primary function of plant landscaping is to create scenery in different seasons with the help of plants and flowers growing in different seasons, such as building a vibrant spring with pear trees, willow trees, etc. and a beauty of summer with lotus. At the same time, choosing some plants with less seasonal change, such as pine and cypress, can create a stable image, so that people can experience the beauty of the garden throughout the year ${ }^{[2]}$. Another major function of plant landscaping is to construct special landscape and outdoor space. In many gardens, one landscape after another is built mostly, which makes the garden a strong variety and appreciation but the construction work is relatively large. However, plant landscaping in conjunction with buildings can often lead to a quiet, natural and harmonious state with the effect of plants on buildings, so that people living in cities can temporarily get out of the hustle and bustle to gain emotional experience in the soul.

\section{Present Situation of Contemporary Plant Landscaping in China}

The long history of thousands of years has witnessed the development and progress of the garden 
art. At the same time, because of its own aesthetic and artistic characteristics, the plant landscaping is developing in full swing, and have also shown great achievements in the current urban planning. Since the founding of PRC, with the rapid development of society and the continuous improvement of people's living standards, more attention has been paid to environmental needs after basic material satisfied ${ }^{[3]}$ In urban planning and construction, plants are often used to create comfortable and beautiful seep environment to improve the quality of surrounding environment and life through the construction of ecological gardens, so as to distance away from the countryside and be close to nature. After the reform and opening up, attention is paid to the use of natural plants rich in form and color changes to create landscape in the planning and construction of urban parks, giving the parks more outstanding artistry and natural vitality. Since the 1990s, the concept of constructing ecological gardens has been more emphasized in our country, which has been fully shown in the "Pearl Garden" in Shanghai where good ecological benefits has been made with the help of the plant landscaping. It lays a good foundation for the construction of ecological gardens.

Plant landscaping creates a good living environment for people and in the meantime, people can be devoted to aesthetic enjoyment in the experience of beauty. The plants in the gardens grow vigorously, which brings people not only aesthetic enjoyment, but also a kind of spiritual infection ${ }^{[4]}$. In recent years, the Bureau of Land and Resources and the local tourism bureaus have attached increasingly importance to plant landscaping and have increased the investment for urban forest parks, thus promoting the formation of plant communities and protecting plant diversity. The construction of ecological gardens also promotes the development of regional tourism and local economy, and improves the quality of life of residents in cities. Plant landscaping can also achieve the effect of beautifying the environment, and absorb carbon dioxide and dust in the air with the help of plant photosynthesis and adsorption to increase oxygen content and reduce the dust in the air, providing people with a beautiful living environment, as a result, people's happiness is enhanced.

In addition, all departments and personnel actively participate in the plant landscaping at present to create a good urban living environment together, displaying the more significance of the function of the plant landscaping. But from perspective of the actual effect, our plant landscaping needs to be improved compared with some countries with perfect ecological garden construction, the concrete questions are shown in the following several aspects: (1)China has a vast territory and a very rich variety of plants, while few plant varieties are used in landscaping. Only 400 or 500 species of plants are applied in the landscaping, and merely dozens of them are selected for the plant landscaping in many cases, causing single landscape with homogenization, to has a huge impact on the aesthetic value of the gardens obviously ${ }^{[5]}$. In addition, the overall number of plants is not up to ten thousand frequently in the plant landscaping, resulting unreasonable overall allocation of garden plants. (2)Under the influence of poor ideas and low design level, in many cases, many designers for plant landscaping in our country cannot improve their ability to develop the art of plant landscaping, and cannot cultivate a variety of plants on the whole. For example, our country has a lot of beautiful flower plants often abandoned by landscape artists ignoring the value of all kinds of plants in the actual plant landscaping, which is obviously a waste of natural plants. Therefore, in view of the present development of plant landscaping in China, it is necessary to constantly excavate the value of plants, in order to ensure that more plants can be introduced in the plant landscaping, then creating more landscape with abundant plant species for the society.

\section{Development Trend of Plant Landscaping}

Summarizing the characteristics of plant landscaping design all over the world, a very strong human culture is shown, which highlights the development trend of humanization in future plant landscaping. The humanized characteristics in plant landscaping are developed according to people's needs for appreciation and living. ${ }^{[6]}$ With the rapid development of social economy, people's consciousness and concept have changed greatly. Current education, medical treatment, shopping and other fields haven stood on humanization, hoping to meet people's needs with the help of humanized concept. In order to survive in the current economic and social environment, the plant landscaping must closely revolve around the demand of humanization, and the selection and design 
of plants should fully embody the characteristics of humanization, so as to show the value and significance of plant landscaping, thus providing a beautiful experience and enjoyment for people's life and development. Humanized plant landscaping meets people's psychological and physiological needs, drawing people closer to nature and return to natural origin, thus relaxing people's minds and improving people's conviction. Therefore, it is necessary to follow the development trend of humanization in the future plant landscaping.

At present, the ecological concept has been deeply rooted in the minds of the people, which also emphasizes the needs to follow the ecological principles in the plant landscaping, where trees should be planted in correspondence appropriate fields to cultivate the plants well to show their native color and charm. In the selection of plants, we need to know the environmental conditions of the original place where these plants grow and the growth trend of the trees. For example, if cinnamomum camphora, palms, and nandina domestica growing in the south with poor cold resistance are desired to plant in the north, the leeward and sunny places should be considered at the very beginning of the planting; Amur honeysuckle, wintersweet, ivy and other plants like living without the sun, so they are generally planted in the shade ${ }^{[7]}$. And in order to ensure the survival rate of plants, plants easy to survive and bearing trim with long life and less pests and diseases should be chose as far as possible, such as cedar, ginkgo biloba, metasequoia, platanus, photinia serrulate, hydrangea macrophylla, Iris, tradescantia virginiana, etc. In the layout of the plants, local plants and exotic plants should match each other to create a unique landscape and improve the ornamental value of plant landscape. At the same time, based on ecological principles, plant landscaping should make every effort to allocate multi-levelled, multi-structural and multi-functional plant communities, in order to achieve the unity of ecological beauty, color beauty and artistic beauty, resulting in creating clean, civilized and modern landscape. In addition, under the concept of ecology, the plant landscape construction is gradually integrated into people's living space to advocate the application of three-dimensional models in residential areas, such as plant climbing plants on walls to weaken the rigid geometric lines of architectural forms, so as to beautify and color the space to significantly show the ecological benefits of residential areas, thus promoting the ecological development of plant landscaping.

The artistry of plant landscape is the basis of its aesthetic value. When plant landscape is built, reasonable design should be made according to the local geological conditions, natural environment and the overall architectural modeling. For example, the artistic nature of the planning of the campus plant landscaping reflects in the allocation of plants ${ }^{[8]}$. Specifically, metasequoia can be planted on both sides of the school gate to show its majesty, and different colors of flowers can be cultivated around to add the beauty; On both sides of the road, adumbral trees can be planted, for example, camphor trees may be chose in the south to form a tree-lined path; Hydrophilic plants, the most common of which is willow can be planted near the pool in which ornamental lotus can be planted. Different plants are cultivated in different places to give different visual enjoyment to the people and unique images to architectures. Besides, in the artistic plant landscaping, plants adapt to the local environment should be selected as far as possible by the consideration of the integration of plants with natural climate and architecture, to highlight the artistic connotation of the plant landscaping through reasonable plant selection.

\section{Conclusion}

In conclusion, the local plant landscaping has been gradually improved after years of development, and has made remarkable achievements. As a kind of garden art, plant landscaping not only provides people with aesthetic enjoyment, but also promotes economic development. Considering that some problems still exist in the contemporary plant landscaping in China, it is necessary to further grasp the development trend of plant landscaping, so as tp create rich and colorful landscape, thus to promote the development of plant landscaping in China. 


\section{References}

[1] Wei Daiqing. Status and Trend of Plant Landscaping Setup and Application in Guiyang City[J]. Modern Horticulture, 2018,15(4).

[2] Zhou Hanhan. Characteristics, Strategies and Trends of Modern Plant Landscaping[J]. Modern Horticulture, 2018,22(1):104-105.

[3] Liu Can'gang, and Tian Hui. On the Development Trend of Plant Landscape Design[J]. Industry C, 2016,14(9):00275-00276.

[4] Hu Fang. Advances in Evaluation of Plant Landscaping[J]. Engineering Technology: Citation, 2016,23(5):00231-00232.

[5] Yan Yunshan. A Brief Analysis on the Trend of Modern Plant Landscaping Allocation in China [J]. Building Materials and Decoration, 2016,6(13).

[6] Xing Hongtao, Liu Haibo, and Huang Jinfeng. The Present Situation and Measures of Plant Landscaping of Road in Northern Cities of Jiangsu Province - A Case Study of Road Greening in Xuzhou City [J]. Modern Horticulture, 2016,14(2):116-118.

[7] Pei Yuanzheng. Advances in Evaluation of Plant Landscaping[J]. Friends of Peasants, 2017,8(10):146-146.

[8] Shan Xiaorong. Research and Analysis on Space Design of Plant Landscape[J]. Engineering Technology: Abstract, 2016,14(9):00158-00158. 